\title{
A Web-Based Intelligibility Evaluation of Sign Language Video Transmitted at Low Frame Rates and Bitrates
}

\author{
Jessica J. Tran ${ }^{1}$, Rafael Rodriguez ${ }^{1}$, Eve A. Riskin ${ }^{1}$, Jacob O. Wobbrock ${ }^{2}$ \\ ${ }^{1}$ Electrical Engineering \\ DUB Group \\ University of Washington \\ Seattle, WA 98195 USA \\ \{jjtran, rodriraf, riskin\}@uw.edu \\ ${ }^{2}$ The Information School \\ DUB Group \\ University of Washington \\ Seattle, WA 98195 USA \\ wobbrock@uw.edu
}

\begin{abstract}
Mobile sign language video conversations can become unintelligible due to high video transmission rates causing network congestion and delayed video. In an effort to understand how much sign language video quality can be sacrificed, we evaluated the perceived lower limits of intelligible sign language video transmitted at four low frame rates $(1,5,10$, and 15 frames per second [fps]) and four low fixed bitrates (15, 30, 60, and 120 kilobits per second [kbps]). We discovered an "intelligibility ceiling effect" where increasing the frame rate above $10 \mathrm{fps}$ decreased perceived intelligibility, and increasing the bitrate above $60 \mathrm{kbps}$ produced diminishing returns. Additional findings suggest that relaxing the recommended international video transmission rate, $25 \mathrm{fps}$ at $100 \mathrm{kbps}$ or higher, would still provide intelligible content while considering network resources and bandwidth consumption. As part of this work, we developed the Human Signal Intelligibility Model, a new conceptual model useful for informing evaluations of video intelligibility.
\end{abstract}

\section{Categories and Subject Descriptors}

K.4.2. [Social Issues]: Assistive technologies for persons with disabilities; H.5.1 [Information Interfaces and Presentation]: Multimedia Information Systems - Video.

\section{General Terms}

Performance, Experimentation, Human Factors.

\section{Keywords}

Intelligibility, comprehension, American Sign Language, bitrate, frame rate, video compression, web-survey, communication model, Deaf community.

\section{INTRODUCTION}

Real-time mobile video communication allows deaf and hard-ofhearing people to communicate in their native language. American Sign Language (ASL) is signed in the United States (U.S.) and is a visual language with unique grammar and syntax independent of spoken languages. U.S. cellular networks do not provide unlimited data plans and may throttle networks speeds to high data rate consumers [34]. The high video transmission rates implemented by commercial mobile video applications place a heavy load on the total available network bandwidth. They cause

Permission to make digital or hard copies of all or part of this work for personal or classroom use is granted without fee provided that copies are not made or distributed for profit or commercial advantage and that copies bear this notice and the full citation on the first page. To copy otherwise, or republish, to post on servers or to redistribute to lists, requires prior specific permission and/or a fee.

Conference'10, Month 1-2, 2010, City, State, Country.

Copyright 2010 ACM 1-58113-000-0/00/0010 ...\$15.00.

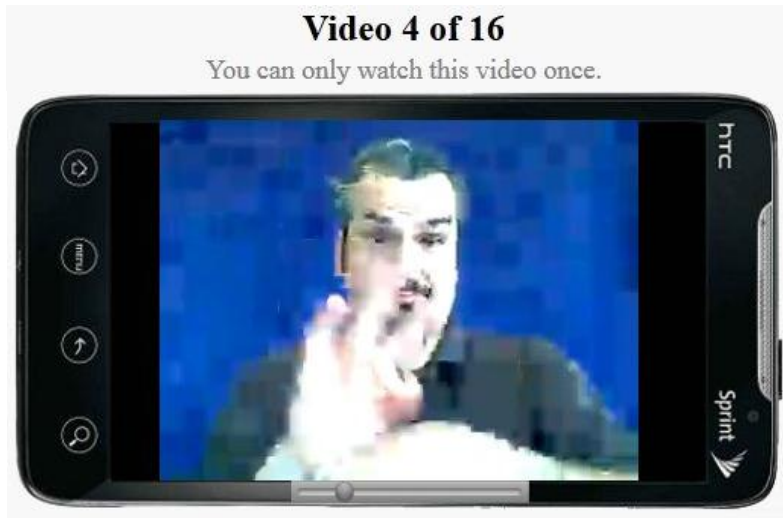

Q1) How easy was the video to understand?

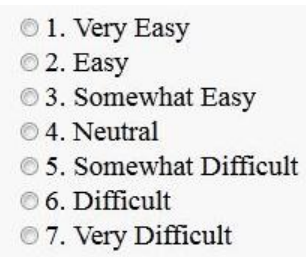

Figure 1: Screen short of one video from web survey evaluating intelligibility of sign language video displayed at $\mathbf{1 5}$ frames per second at $\mathbf{3 0}$ kilobits per second.

network congestion and delayed video, often interrupting mobile sign language video conversations. The current sign language video transmission rate recommended by the Telecommunication Standardization Sector (ITU-T) Q.26/16 is 25 frames per second (fps) at 100 kilobits per second (kbps) or higher displayed at $352 \times 288$ pixels [21]. Our research demonstrates that intelligible sign language video communication can result at frame rates and bitrates less than recommended by the ITU-T.

In our evaluation of mobile sign language video intelligibility, we discovered a lack of uniformity in the way that signal intelligibility and signal comprehension are operationalized in human-centered evaluations. We introduce a new model, the Human Signal Intelligibility Model (HSIM), to distinguish the components comprising video intelligibility from the components comprising objective video quality and video comprehension. Intelligibility is defined as the capability of a signal to be understood, given that the signal was clearly articulated, captured, transmitted, received, and perceived by the receiver, including the environmental conditions affecting these steps. Comprehension is defined as signal intelligibility plus the receiver having the prerequisite knowledge to understand the information. Both intelligibility and comprehension are human-centered concepts, unlike objective video quality measures such as peak signal-to- 
noise ratio (PSNR). Our web study uses the HSIM to evaluate video intelligibility as distinct from objective video quality or comprehension.

We created a national web survey, as shown in Figure 1, evaluating the lower limits of intelligible sign language video intended to be viewed on small mobile devices. The web survey had 99 respondents watch 16 short ASL videos of a male native ASL signer signing short sentences shown at four low frame rates $(1,5,10$, and $15 \mathrm{fps})$ at four low fixed bitrates $(15,30,60$, and $120 \mathrm{kbps})$ in a full factorial design. We discovered that videos transmitted at $10 \mathrm{fps}$, independent of bitrate, received the highest mean Likert scores for intelligibility ( $M=5.09$, std. error=.08). Responses were based on a 7-point Likert scale ranging from 1strongly disagree to 7-strongly agree. Surprisingly, we found an "intelligibility ceiling effect" where ASL video transmitted at 15 fps, independent of bitrate, reduced perceived intelligibility of ASL video $(\mathrm{F}(1,1139)=77.22, p<.0001)$. This particular finding suggests transmitting sign language video at frame rates higher than $10 \mathrm{fps}$ is not necessary to provide intelligible content, especially when network resources like bandwidth need to be preserved. We also discovered that videos transmitted at $60 \mathrm{kbps}$ vs. $120 \mathrm{kbps}$ were not perceived to increase intelligibility $(\mathrm{F}(1,1139)=4.62$, n.s. $)$. These and other findings suggest that intelligible sign language video can be transmitted at $10 \mathrm{fps}$ at 60 $\mathrm{kbps}$, which is lower than the recommended ITU-T standards.

\section{RELATED WORK}

The effects of frame rate and bitrate reductions on objective video quality have been widely researched for sign language learning and comprehension, evaluating subjective video quality, creating video quality measures, and evaluating video intelligibility. However, unlike the present work, none of this prior work has been intended for facilitating real-time mobile sign language conversations or considering the bandwidth needed to support such communication. Our works fills this gap by identifying the lower limits of intelligible mobile sign language video.

\subsection{Sign Language Learning \& Comprehension}

Sign language learning is more nuanced than holding sign language conversations because linguistic accuracy is most important. Therefore, the effect of frame rate reduction on sign language learning has been extensively researched [5,11,12,23]. Johnson and Caird [12] investigated whether perceptual ASL learning was affected by video transmitted at $1,5,15$, and $30 \mathrm{fps}$. In a discrimination task, participants made a yes-no decision about whether the displayed sign and the English word shown matched. They found that frame rates as low as $1 \mathrm{fps}$ and $5 \mathrm{fps}$ were sufficient for novice ASL learners to recognize learned ASL gestures. In our work, gesture recognition is not enough to support meaningful conversations; therefore, we investigate the impact low frame rates and low bitrates have on sign language sentences.

Hooper et al. [11] define comprehension as the ability for respondents to accurately retell stories verbatim. They investigated the impact on ASL learning comprehension when ASL video was presented at 6,12 , and $18 \mathrm{fps}$ and displayed at $240 \times 180,320 \times 240$, and $480 \times 360$ pixels at $700 \mathrm{kbps}$. Hooper et al. found display size did not affect comprehension, but varying frame rates did. Students performed better after viewing video at $12 \mathrm{fps}$ than at $6 \mathrm{fps}$, and at $18 \mathrm{fps}$ than at $6 \mathrm{fps}$; however, there was not a significant difference in performance between $18 \mathrm{fps} v s$. $12 \mathrm{fps}$.
Sperling et al. [23] defines intelligibility as the ability to correctly recognize signs. They investigated ASL video intelligibility transmitted at 10,15 , and $30 \mathrm{fps}$ displayed at $96 \times 64,48 \times 32$, and $24 \times 16$ pixels, while applying a grayscale image transformation. They found that common isolated ASL signs shown at $96 \times 64$ pixels at $15 \mathrm{fps}$ and $30 \mathrm{fps}$ did not have a noticeable difference in intelligibility, but lowering the frame rate to $10 \mathrm{fps}$ did. While prior work showed that lower frame rates can impact isolated sign recognition, these results may not hold true for mobile sign language video conversations. Our work goes beyond sign recognition and investigates video intelligibility to support twoway conversations.

\subsection{Subjective Video Quality}

We aim to discover whether frame rate or bitrate has more impact on ASL video intelligibility. A subjective experiment, conducted by Yadavalli et al. [32], evaluated frame rate preferences passively viewed for low, medium, and high motion sequences displayed at $352 \times 240$ pixels; three frame rates $(10,15$, and 30 $\mathrm{fps})$; and three bitrates (100, 200, and $300 \mathrm{kbps})$. Viewers preferred video at $15 \mathrm{fps}$ across all bitrates and video sequences, which suggest that 15 fps represents a compromise rate between frame and motion quality. At $300 \mathrm{kbps}$, respondents preferred video at $30 \mathrm{fps}$, suggesting that motion quality is more important once adequate frame quality is achieved. Like Yadavalli et al.'s work, we aim to determine whether ASL video becomes more intelligible by increasing the frame rate once frame quality (determined by bitrate) is adequate. But unlike this prior work, we require respondents to actively watch and understand ASL video content.

Masry and Hemami [15] evaluated subjective video quality perception of non-ASL streaming video content transmitted at 10 , 15 , and 30 fps and six bitrates $(40,100,200,300,600$, and 800 Kbps). Respondents viewed fifteen 30 -second video clips consisting of low, medium, and high motion sequences. After each video, respondents rated video quality on a slider ranging from 0 (worst) to 100 (best). The researchers found that respondents favored video shown at $15 \mathrm{fps}$ over $10 \mathrm{fps}$ when shown at a fixed bitrate. Cavender et al. [4] used ASL video clips and also discovered a frame rate preference for viewing ASL video at a fixed bitrate. They evaluated intelligibility of ASL video displayed at different frame rates (10 and $15 \mathrm{fps})$, bitrates (15, 20, and $25 \mathrm{kbps}$ ), and region-of-interest (ROI) encoding levels (0, -6 , and -12 ROI). (The ROI was an approximation of where the signers face and hands were located.) Our work investigated more frame rates and bitrates than Cavender et al., and our findings corroborate the finding that respondents rated higher intelligibility for video viewed at $10 \mathrm{fps}$ over $15 \mathrm{fps}$ at a constant low bitrate, which is opposite of what Masry and Hemami found.

The findings from our work and elsewhere $[11,12,23]$ suggest that there is a threshold above which increasing the frame rate does not significantly improve video intelligibility. Our research builds upon Cavender et al.'s [4] findings and more rigorously investigates intelligibility of sign language video. Cavender et al.'s laboratory study used prerecorded video filmed with a stationary video camera, which allowed more space in the signing region. By contrast, the videos evaluated in our web study were representative of the angle and signing space constrained by mobile devices. Also, our research goal was to discover how much video quality could be reduced before sign language intelligibility was compromised, a goal not approached by Cavender et al.'s work. 


\subsection{Objective Video Quality Measures}

Measuring subjective video quality is time consuming, contentspecific, and requires many subjects to produce generalizable findings. By contrast, peak signal-to-noise ratio (PSNR) is commonly used in video compression to measure objective video quality after lossy compression [29]. However, PSNR has been shown to not always accurately represent humans' subjective judgments about video quality [7,17,24,26,28]. Numerous researchers have attempted to map PSNR to subjective responses by creating new objective video quality perception metrics $[19,27,30,33]$; however, these objective measures have been content-dependent.

Content intelligibility is most important for sign language video; therefore, objective video evaluations are not the most appropriate way to characterize video quality. Ciaramello and Hemami [6] recognized that sign language video needs to be evaluated in terms of subjective intelligibility. They created a computational model of intelligibility for ASL called CIM-ASL, which measures the perceptual distortions of video regions deemed important for conveying information, specifically the hands, face, and torso of a signer. The CIM-ASL model has been shown to have statistically significant improvements over PSNR when estimating distortions in the CIM-ASL-defined signing region. However, the CIM-ASL model relies on video quality perception with the assumption that greater video quality in the signing region leads to higher intelligibility. By contrast, our model of subjective intelligibility for sign language video goes beyond measuring video objectively and details the components impacting subjective sign language intelligibility.

\subsection{Defining Intelligibility}

Often, intelligibility and comprehension are loosely defined and used interchangeably in evaluations of video quality. Some researchers focused on measuring signal intelligibility with the intent that if one finds the signal intelligible, then comprehension of content follows $[1,8,9,11,18]$. In his famous work, Shannon [22] created a simple abstraction for communication called the channel, which consists of a sender (the information source), a transmission medium with noise and distortion, and a receiver. However, the channel model only focuses on the communication channel itself without considering the surrounding environment or properties of a human sender and receiver. Existing communication models $[2,3]$ that attempt to distinguish intelligibility from comprehension are poorly defined. Berlo [3] created the source, message, channel, receiver (SMCR) model of communication, which consists of twenty different elements but does not clearly identify which elements produce intelligible communication. Barnlund [2] proposed a transactional model of communication suggesting individuals are simultaneously engaging in the sending and receiving of messages. Although Barnlund's model represents how information is transferred, it does not attempt to distinguish intelligibility from comprehension.

We believe that signal intelligibility and signal comprehension need to be distinguished. Intelligibility depends on signal quality, specifically how the signal was captured, transmitted, received, and perceived by the receiver, including the environmental conditions affecting these steps. Comprehension relies on signal intelligibility and the human receiver having the prerequisite knowledge to understand the information. These insights lead us to propose our own intelligibility model, described next.

\section{Human Signal Intelligibility Model}

We present the Human Signal Intelligibility Model (HSIM) to address the lack of uniformity in the way that signal intelligibility and signal comprehension have been operationalized, especially in contrast to objective video quality measures. This model distinguishes subjective video intelligibility from objective video quality and video comprehension, which we argue are three usefully distinct and separable things.

The HSIM (1) extends Shannon's theory of communication [22] to include the human and environmental influences on signal intelligibility and signal comprehension, and (2) identifies the components that make up the intelligibility of a communication signal, separating those from the comprehension of a communication signal. Signal intelligibility and signal comprehension are separable concepts because an intelligible signal does not entail comprehension by a receiver lacking the requisite knowledge for understanding.

We claim that the capability of a signal (e.g., video) to be comprehended is different than whether a signal is actually comprehended in any given instance, and this capability is the intelligibility of a signal. In the case of sign language video, intelligibility is affected by the human articulation of the signal; the environment affecting that articulation; the channel capturing, transmitting, receiving, and portraying that signal (the items in Shannon's model); the human perception of that signal; and the environment affecting that perception all affect intelligibility. Figure 2 shows a block diagram illustrating the components comprising intelligibility within the HSIM.

Whether or not the signal is actually understood involves all of the components comprising intelligibility and one additional component, namely the knowledge of the human receiver being adequate to understand the information; that is, to make sense of it. Because whether or not the signal is understood by the receiver is a part of the signal's ability to be comprehended, the receiver's mind is included in the components comprising comprehension in Figure 2. The knowledge of the human sender is irrelevant to comprehension by the receiver. For example, the sender could be a robot articulating ASL signs, but having no knowledge of ASL. Our definition of signal intelligibility and signal comprehension builds upon Koul's definition of speech signal quality. Koul [13]

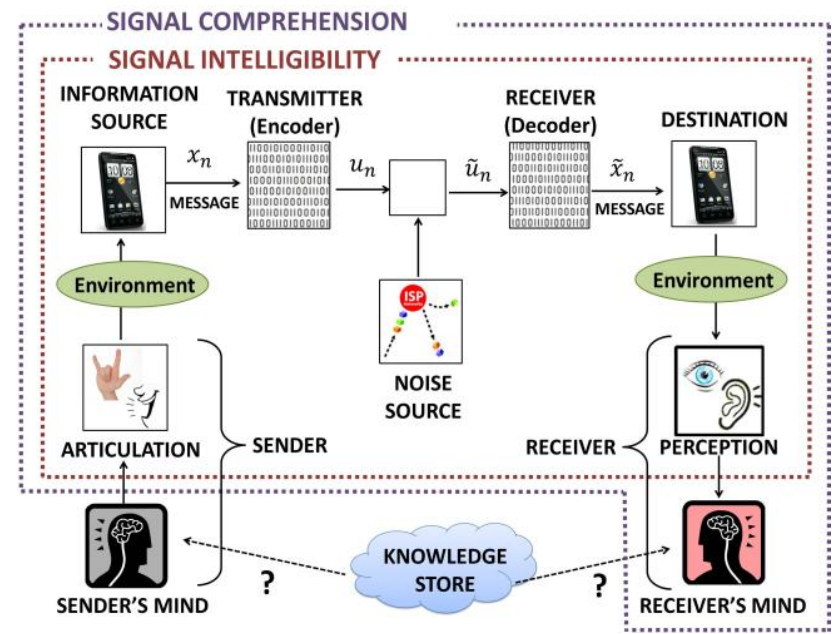

Figure 2: Block diagram of the Human Signal Intelligibility Model. Note that the components comprising signal intelligibility are a subset of signal comprehension, which is signal intelligibility plus the receiver's mind. 
defines intelligibility of a speech signal as the individual's ability to recognize phonemes and words presented in isolation. Comprehension is defined as the listener's ability to process the linguistic message as a whole.

Our HSIM goes beyond Koul to include environmental influences in which a signal is transmitted and received. Lighting is an example of an environmental factor that may influence signal intelligibility. For instance, viewing sign language video on a mobile device outside on a sunny day would make the screen appear dark. This environmental factor would clearly affect the ability for the video to be perceived by the receiver, compromising its intelligibility. (By contrast, the video's objective quality (PSNR) would be unaffected by sunny outdoor conditions.) Recognizing that the environment can influence signal intelligibility is why the environment is included in the HSIM block diagram.

The HSIM also explicitly separates the sender into two parts, the sender's mind and the sender's articulation. Similarly, the HSIM separates the receiver into two parts, the receiver's mind and the receiver's perception. The sender's articulation impacts intelligibility and comprehension because for sign language video, the quality in which information is conveyed influences the receiver's ability to receive the content. For example, a fluent ASL signer could have a motor impairment that would limit their ability to sign clearly. The physical limitation impacts the sender's signal articulation, which impacts the intelligibility of that signal for the receiver.

The receiver's perception also influences his or her ability to process information. For instance, the sender could sign perfectly clear ASL, but if the receiver has low vision, the signal would be unintelligible to that receiver. However, since the sign language video was clearly signed, it may be intelligible to other receivers. These and other examples illustrate the importance of recognizing human factors and environmental influences on signal intelligibility and signal comprehension. Intelligibility, then, is inherently a contextualized concept, unlike objective signal quality as measured by PSNR.

The HSIM reveals an important fact about signal intelligibility: it cannot be measured directly, as the ability to be comprehended cannot be easily separated from the actual comprehension of a signal. Fortunately, intelligibility can be inferred by measuring signal comprehension in the presence of fully capable receivers' minds with more than adequate linguistic knowledge to understand the signals they receive. Such minds remove any chance that a lack of knowledge will affect comprehension, leaving only intelligibility to explain any comprehension difficulties.

\section{WEB SURVEY DESIGN}

The HSIM informs the design of our web study evaluating how much frame rate and bitrate can be reduced before intelligibility is compromised in sign language video. Owing to the need to ensure all receivers' minds are fully capable of comprehension, we screen participants for ASL fluency. Thereafter, we can attribute differences in comprehension to differences in intelligibility and not knowledge.

Our web study evaluated sign language video intelligibility transmitted at four low frame rates $(1,5,10$, and $15 \mathrm{fps})$ and four low bitrates $(15,30,60$, and $120 \mathrm{kbps})$ in a full factorial design. The web study was selected over a laboratory study because parameter settings could be evaluated with participants from across the nation. A mobile web survey was considered, but at the time of web development, we found too much variability across mobile devices and mobile web browsers, which we could not control as an environmental influence.

The survey consisted of three parts and took 12-26 minutes per respondent to complete. Part 1 had two practice videos to allow familiarization with the survey layout. Part 2 was the survey evaluating intelligibility of 16 different videos shown in a singlestimulus experiment. Part 3 asked demographic questions. Upon survey completion, participants had an opportunity to enter their email for a chance to win one of four $\$ 75$ gift cards. Their e-mail was not associated with their anonymous and confidential responses.

The web survey began by asking participants to self-report their fluency in ASL. ASL interpretations of the English text instructions were shown side-by-side throughout the web survey to increase accessibility. A professional ASL interpreter, who is a child of deaf adults, was consulted before filming.

\subsection{Video Stimuli}

Users of mobile sign language video communication are limited by the front facing camera angle and confined signing space. Since the web survey would display pre-recorded video on a computer screen parallel to the participant, the videos used in the survey simulated the 45 degree angle and signing space that would typically be displayed on a small mobile device. An Acer Iconic tablet running Android Honeycomb 3.2.1 was used to record a native male ASL signer/consultant, signing 16 short ASL sentences that included various amounts of finger spelling and descriptive lexicons. The ASL signer was asked to sign slowly and sign all signs within the allowable signing space. The ASL signer sat in front of a solid dark blue background. Video length ranged from 15-30 seconds. At the time of video recording, the front facing camera of smartphones, like Sprint's EVO phone, only recorded compressed video in $3 \mathrm{GP}$ file format. Recording video from a smartphone was not an option due to added video compression. The tablet recorded uncompressed video in 4:2:2 YUV format at $25 \mathrm{fps}, 8.73 \mathrm{Mbps}$ with $320 \times 240$ screen resolution.

The original YUV videos were encoded using the open source H.264 encoder [20]. The encoded videos were converted to MPEG-4 using a publicly available converter [14] that does not contribute additional artifacts. The web survey displayed the videos using Apple's QuickTime media player [35] since no additional artifacts were contributed by this player.

\subsection{Survey Components}

All videos were displayed at $320 \times 240$ pixels in the middle of the computer screen. A picture of the Sprint EVO phone was placed behind each video to simulate the mobile appearance of the videos. Each video was shown once, without the option to repeat or enlarge the video, and then removed from the screen and replaced by two questions shown one at a time. Figure 3 is an example of question 1, which asked respondents to rate their agreement on a 7-point Likert scale with, "How easy was the video to understand?" The 7-point Likert scale was shown in descending vertical order from very easy to very difficult. Figure 4 is an example of a trivial comprehension question pertaining to the video shown. A four point multiple choice answer appeared with a corresponding image.

We unobtrusively logged the time it took to answer the comprehension question to compare if there was any relationship 


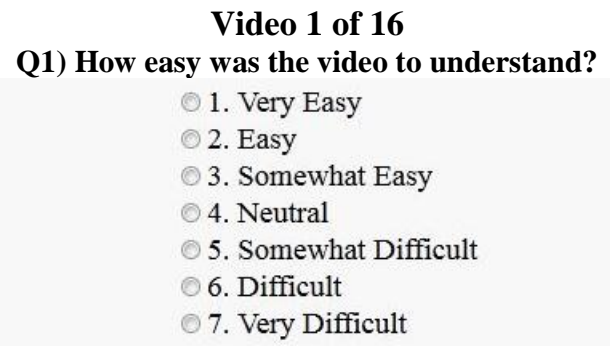

Figure 3: Example of question 1 shown in web survey.

Q2) How does Stephanie get to school?

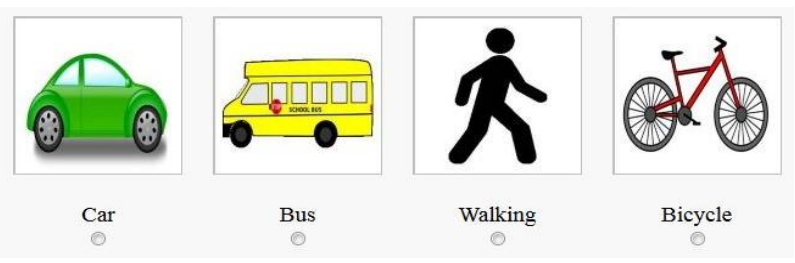

Figure 4: Multiple choice comprehension question example.

between the time to answer the comprehension questions and rating the perceived ease of understanding the video. The start time began when the question appeared on the screen and the stop time occurred once the 'Next' button was clicked. Since the ease of comprehension varied with each video, the comprehension questions were mainly used to confirm that the respondent was paying attention to the video.

The same layout used in part 1 was used in part 2 of the survey in which participants watched 16 different videos at each bitrate and frame rate combination. Videos were randomly displayed using a Latin Square. The frame rate and bitrate settings did not change within each video clip. Finally, the survey concluded with Part 3 asking demographic questions such as: "How many years have you signed ASL?"; "From whom did you learn ASL?"; "Are you deaf or hard-of-hearing?"; and "Are you a native ASL signer?

\section{RESULTS}

Our web survey received 300 hits, with 99 respondents completing the survey, all of whom self-reported fluency in ASL. We eliminated results from those who responded with the same answers for all 16 videos, such as selecting all $1 \mathrm{~s}$ or all $7 \mathrm{~s}$. We analyzed data from 77 respondents (48 women). Their age ranged from $18-72$ years old (median $=40$ years, $S D=12.73$ years). Of the 77 respondents: 56 were deaf (38 indicated ASL as their native language, 11 have parents who are deaf), 54 indicated ASL as their daily language, and the number of years they have spoken ASL ranged from 5-59 years (median=28 years, $S D=12.73$ ). All but 7 respondents own a smartphone and send text messages; 65 indicated they use video chat; and 53 use video relay services.

\subsection{Perceived Intelligibility}

Results will be reported in terms of intelligibility even though comprehension questions were asked. Recall that video intelligibility can be inferred from comprehension questions provided that the receivers' knowledge stores are fully adequate to understand the received signals - in this case, once ASL fluency is established. Nonparametric analyses were used to analyze the Likert responses since the data were ordinal and not normally distributed. Analysis was performed using the nonparametric Aligned Rank Transform [31] procedure that enables the use of ANOVA after alignment and ranking, while preserving interaction effects.

\subsubsection{Frame Rate Main Effect}

Frame rate was found to have a significant main effect on video intelligibility $(\mathrm{F}(3,1139)=636.99, \quad p<.0001)$. Post-hoc contrast tests with Holm's sequential Bonferroni correction [10] were performed for $1 \mathrm{fps} v s$. $5 \mathrm{fps} ; 5 v s .10 \mathrm{fps} ; 5 v s$. $15 \mathrm{fps}$; and $10 \mathrm{fps}$ vs. $15 \mathrm{fps}$. Table 1 and Figure 5 list the mean Likert score for question 1, where higher scores correspond to higher agreement with the ease of perceived understanding of video content. As expected, videos displayed at $5 \mathrm{fps}$ when compared to $1 \mathrm{fps}$ received higher mean Likert scores for video intelligibility $(\mathrm{F}(1,1139)=921.07, p<.0001)$. Videos displayed at $10 \mathrm{fps}$ when compared to $5 \mathrm{fps}$ received higher mean Likert scores for video intelligibility $(\mathrm{F}(1,1139)=111.13, \quad p<.0001)$. However, when comparing $10 \mathrm{fps} v s .15 \mathrm{fps}$, video displayed at $10 \mathrm{fps}$ was found to have a higher mean Likert score for intelligible content $(\mathrm{F}(1,1139)=77.22, p<.0001)$. As Figure 5 shows, video displayed at $10 \mathrm{fps}$ (averaged across four bitrates) received higher mean Likert scores than all other frame rates. An unexpected finding was that video was not perceived to be more intelligible at $5 \mathrm{fps}$ vs. $15 \mathrm{fps}(\mathrm{F}(1,1139)=3.11$, n.s. $))$. One would expect that a higher frame rate would yield higher intelligibility for a temporal language since the ITU-T recommends $25 \mathrm{fps}$ for intelligible sign language video.

\subsubsection{Bitrate Main Effect}

Changing the bitrate was found to have a significant main effect on intelligibility of ASL video $(\mathrm{F}(3,1139)=145.53, p<.0001)$. Post-hoc contrast tests with Holm's sequential Bonferroni correction were performed for $15 \mathrm{kbps} v s$. $30 \mathrm{kbps} ; 30 \mathrm{kbps} v s .60$ kbps; and $60 \mathrm{kbps} v s$. $120 \mathrm{kbps}$. Unsurprisingly, increasing the bitrate from $15 \mathrm{kbps}$ to $30 \mathrm{kbps}$ to $60 \mathrm{kbps}$ was found to significantly improve ASL video intelligibility $(\mathrm{F}(1,1139)=82.75$, $p<.0001)$. However, videos displayed at $60 \mathrm{kbps} v s .120 \mathrm{kbps}$ were not found to be significantly different in terms of intelligibility $(\mathrm{F}(1,1139)=4.62$, n.s. $)$.

\subsubsection{Frame Rate $\times$ Bitrate Interaction}

There was also significant frame rate $\times$ bitrate interaction $(\mathrm{F}(9,1139)=23.40, \quad p<.0001)$. Upon closer inspection, videos transmitted at $10 \mathrm{fps}$, independent of bitrate, received the highest mean Likert scores for ease of understanding video quality as shown in Table 1 and Figure 5. Additionally, videos displayed at $60 \mathrm{kbps} v s .120 \mathrm{kbps}$ were not found significantly different in terms of intelligibility, which is reflected by similar mean Likert scores suggesting that $60 \mathrm{kbps}$ is a high enough bitrate to transmit intelligible video. Video displayed at $1 \mathrm{fps}$ received the lowest mean Likert score, suggesting that $1 \mathrm{fps}$ is too low to support intelligible sign language video conversations.

\subsection{Comprehension Questions}

We unobtrusively logged the time it took participants to respond to the comprehension questions. The logged time started when the question appeared on the screen and ended when the answer was submitted. Thirteen of 16 comprehension questions were answered correctly with $95 \%$ accuracy or higher. We report findings on correctly answered comprehension questions across frame rates (averaged over all four bitrates) and across bitrates (averaged over all four frame rates). Table 2 lists the mean time and standard deviation for respondents who answered the comprehension question correctly.

We discovered that the fastest mean response times for correctly answering the comprehension questions for both frame rate (averaged over all four bitrates) and bitrate (averaged over all four frame rates) also received the highest mean Likert scores for 
Table 1: Mean Likert score responses for ease of understanding video quality. Note higher Likert scores correspond to higher perceived intelligibility.

\begin{tabular}{|c|c|c|c|c|c|c|c|c|}
\hline \multirow{4}{*}{$\begin{array}{l}\text { frame rate } \\
\text { (fps) }\end{array}$} & \multicolumn{8}{|c|}{ Bitrate (kbps) } \\
\hline & \multicolumn{2}{|c|}{15} & \multicolumn{2}{|c|}{30} & \multicolumn{2}{|c|}{60} & \multicolumn{2}{|c|}{120} \\
\hline & Mean & & Mean & & Mean & & Mean & \\
\hline & Likert & std. error & Likert & std. error & Likert & std. error & Likert & std. error \\
\hline 1 & 2.14 & 0.14 & 1.13 & 0.07 & 1.75 & 0.11 & 1.90 & 0.10 \\
\hline 5 & 3.01 & 0.16 & 4.43 & 0.15 & 4.95 & 0.14 & 4.75 & 0.13 \\
\hline 10 & 4.04 & 0.16 & 4.74 & 0.13 & 5.66 & 0.13 & 5.91 & 0.14 \\
\hline 15 & 3.51 & 0.17 & 3.97 & 0.15 & 5.13 & 0.15 & 5.25 & 0.14 \\
\hline
\end{tabular}

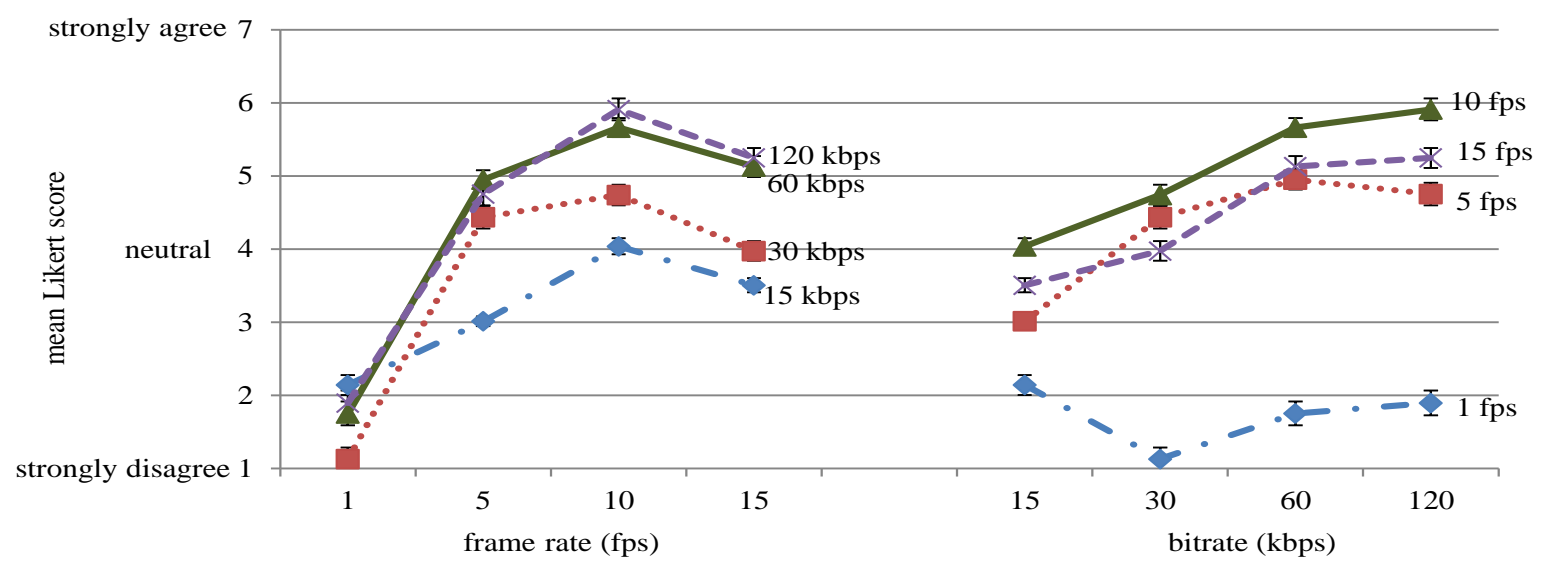

Figure 5: Plot of 7-point Likert ratings for participants' ease of understanding the video for each frame rate and bitrate averaged over all participants. Error bars represent \pm 1 standard error.

Table 2: Mean Likert score (higher values are better) and mean response time (in seconds) for correctly answered comprehension questions for both frame rate (averaged over all four bitrates rates) and bitrate (averaged over all four frame rates). Bold values indicate highest mean Likert scores and fastest times to submit answer.

\begin{tabular}{|c|c|c|c|c|c|c|c|c|c|}
\hline $\begin{array}{c}\text { Frame } \\
\text { rate }(\mathrm{fps})\end{array}$ & $\begin{array}{l}\text { Mean Likert } \\
\text { Score }\end{array}$ & $\begin{array}{l}\text { std. } \\
\text { error }\end{array}$ & $\begin{array}{l}\text { Mean } \\
\text { Response } \\
\text { Time (sec) }\end{array}$ & $\mathrm{SD}$ & $\begin{array}{l}\text { Bitrate } \\
(\mathrm{kbps})\end{array}$ & $\begin{array}{c}\text { Mean Likert } \\
\text { Score }\end{array}$ & $\begin{array}{l}\text { std. } \\
\text { error }\end{array}$ & $\begin{array}{c}\text { Mean } \\
\text { Response } \\
\text { Time (sec) }\end{array}$ & SD \\
\hline 1 & 1.77 & 0.10 & 6.34 & 5.19 & 15 & 3.18 & 0.16 & 5.97 & 3.18 \\
\hline 5 & 4.29 & 0.15 & 6.07 & 3.74 & 30 & 3.61 & 0.13 & 5.81 & 5.28 \\
\hline 10 & 5.09 & 0.14 & 4.19 & 1.74 & 60 & 4.37 & 0.13 & 5.03 & 2.62 \\
\hline 15 & 4.46 & 0.15 & 4.51 & 2.17 & 120 & 4.45 & 0.13 & 4.11 & 1.89 \\
\hline
\end{tabular}

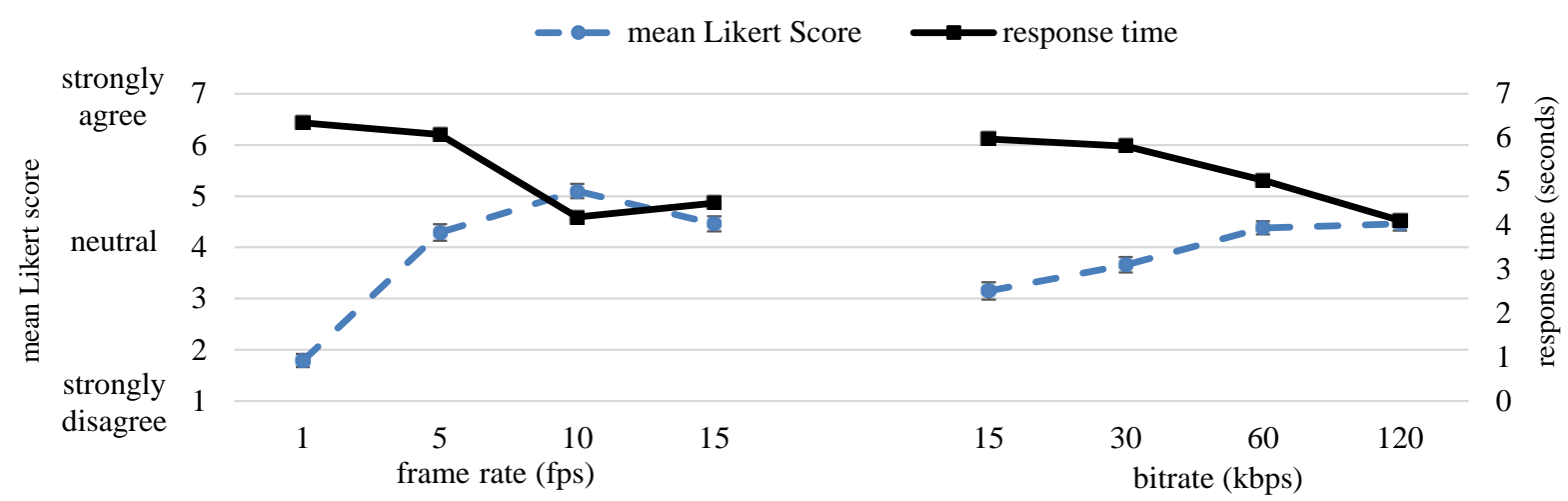

Figure 6: Double $y$-axis plot of a 7-point Likert scale rating participants' ease of understanding the video and mean response time (seconds) for correctly answered comprehension questions for both frame rate (averaged over all four bitrates rates) and bitrate (averaged over all four frame rates). Higher Likert scores correspond to higher perceived intelligibility. 
perceived video intelligibility. These results are demonstrated by the strong negative correlation between mean response time and mean Likert scores for frame rate (averaged overall all four bitrates) $(\mathrm{R}=-0.66)$; and mean response time and mean Likert scores for bitrate (averaged overall all four frame rates) $(\mathrm{R}=-0.82)$. These results suggest that higher perceived video intelligibility leads to faster comprehension of content. Figure 6 is a double $y$ axis plot showing mean Likert score rating perceived video intelligibility $v s$. mean response times for correctly answering the comprehension questions for both frame rate (averaged over all four bitrates) and bitrate (averaged over all four frame rates).

\section{DISCUSSION}

\subsection{HSIM Influence on Study Design}

The HSIM influenced our web study design and which components were held constant. We allowed participants to selfreport ASL fluency to encourage participation. Language fluency questions were asked in the demographic questions to infer levels of ASL fluency. Recall in Section 3, we made the distinction between signal intelligibility and signal comprehension where the latter is defined as signal intelligibility plus human knowledge and the receiver's mind. Since data analysis was performed on data collected from fluent ASL respondents, we were not concerned with language proficiency influencing our results. We controlled the environment in which the video stimulus was recorded and how it was displayed on the web survey. The videos used in the survey were preprocessed to reduce the potential lag time when loading our web survey. We also asked participants to use a high speed internet connection and allow enough time to view all video sequences.

\subsection{Study Findings}

\subsubsection{Frame Rate and Bitrate}

We anticipated finding frame rate and bitrate pairs where video quality begins to affect intelligibility too negatively or diminishing returns begin. Unsurprisingly, respondents overwhelmingly ranked video displayed at $1 \mathrm{fps}$ to have the lowest mean Likert scores for ease of understanding the video content. One fps was selected to achieve a sufficiently low frame rate to observe that intelligibility clearly suffered. Prior work investigating the impact of frame rate on perceived video quality acknowledged not selecting a low enough frame rate to explore in their study $[4,16]$. Although transmitting video at $1 \mathrm{fps}$ is not ideal for ASL conversations, we did notice that transmitting video at 1 fps and $15 \mathrm{kbps}$, which is the lowest bitrate, received the highest mean Likert score across all bitrates at $1 \mathrm{fps}$. This finding corroborates our earlier finding in [25] that people perceived the least amount of negative effects when the lowest frame rate and bitrate settings were applied.

We discovered diminishing returns for videos displayed at 60 kbps and $120 \mathrm{kbps}$ independent of frame rate. Figure 5 shows how the mean Likert scores for $60 \mathrm{kbps}$ and $120 \mathrm{kbps}$, when averaged over all four frame rates, had similar Likert scores and were not found significantly different in terms of intelligibility $(\mathrm{F}(1,1139)=0.47$, n.s. $)$. Our findings suggest $60 \mathrm{kbps}$ is high enough to provide intelligible video conversations.

Another important finding was that video transmitted at $10 \mathrm{fps}$ received a higher mean Likert score than video transmitted at 15 fps across all bitrates. One would think that ASL, which is a temporal visual language, would require video communication to be transmitted at high frame rates; however, we discovered this is not the case at low bitrates. The preference of viewing ASL video at $10 \mathrm{fps}$ over $15 \mathrm{fps}$ was also discovered in earlier ASL video communication research conducted by Cavender et al. [4] However, their findings only reported a slight but significant main effect that frame rate influenced video intelligibility. Our results strongly affirm that ASL video intelligibility peaks at $10 \mathrm{fps}$ across all bitrates. At a fixed low bitrate, more bits are allocated per frame at $10 \mathrm{fps} v s .15 \mathrm{fps}$, and this difference is noticeable enough to result in higher intelligibility. Our findings suggest that relaxing the recommended frame rate and bitrate to $10 \mathrm{fps}$ at of 60 kbps will provide intelligible video conversations while reducing total bandwidth consumption to $25 \%$ of what the current recommended standards of $25 \mathrm{fps}$ at $100 \mathrm{kbps}$ or higher consume.

\subsubsection{Comprehension Question Response Time}

The strong inverse correlation between mean Likert scores rating perceived video intelligibility and mean response times for correctly answering comprehension questions for both frame rate (averaged over all four bitrates) and bitrate (averaged over all four frame rates) suggests higher video transmission rates lead to faster comprehension of video content. There are limitations to these preliminary findings since comprehension difficulty level was not controlled for. We recognize some videos may be easier to comprehend than others due to varied amounts of finger spelling and descriptive lexicons used. Nevertheless, we observed respondents answered comprehension questions more quickly when viewing ASL video with higher perceived intelligibility, suggesting that measuring response time may serve as a proxy for measuring video intelligibility, a relationship we aim to explore more rigorously in the future.

\subsubsection{Signing Speed}

The signing speed used in the video stimuli may have contributed to the non-significant intelligibility improvement of video transmitted at $5 \mathrm{fps} v s$. $15 \mathrm{fps}$. Our findings suggest that $5 \mathrm{fps}$ would be sufficient for intelligible video communication. In future work, we will objectively measure how many signs are perceived by the viewer at $5 \mathrm{fps} v s$. $15 \mathrm{fps}$ to understand the impact of signing speed and frame rate on video intelligibility.

\section{CONCLUSION AND FUTURE WORK}

We presented the Human Signal Intelligibility Model (HSIM) that identifies and distinguishes the components comprising signal intelligibility and signal comprehension. The HSIM informed our web study evaluating the lower limits of sign language video transmitted at four low frame rates and four low bitrates. We find that intelligibility was affected too negatively at $1 \mathrm{fps}$ at $15 \mathrm{kbps}$, and that increasing resources beyond those required for $10 \mathrm{fps}$ at $60 \mathrm{kbps}$ provides negligible gains. Our findings suggest that the recommended ITU-T sign language transmission rates can be relaxed to $10 \mathrm{fps} / 60 \mathrm{kbps}$ while preserving intelligible ASL video and reducing bandwidth and network load.

In future work, we will conduct a laboratory study to evaluate and further demonstrate that intelligible real-time mobile video calls can be made at lower frame rates and bitrates than those recommended by the ITU-T standard. We anticipate the knowledge gained on low video quality intelligibility will make mobile sign language video more accessible and affordable. Finally, we anticipate the HSIM can be used in other signal evaluations of intelligibility and comprehension such as audio and other video streaming media. The knowledge gained about intelligibility of low video quality has the potential to positively influence the user experience of mobile video communication. 


\section{ACKNOWLEDGMENTS}

Thanks to Richard E. Ladner, Gerardo Di Pietro, Jason Smith, John Norberg, Christine Liao, Kyle Rector, Nick Parker, Kristy Winter, and Tobias Cullins for the web study and ASL video feedback; Alex Jansen and Charles Delahunt for help with statistical analyses; Raja Kushalnagar, Lydia Runnels, Jerry Schnepp, Rosalee Wolfe, Christian Vogler, Kathleen Youell, Sorenson VRS, and ZVRS for promoting the web survey; and our respondents. This work was funded in part by Google.

\section{REFERENCES}

[1] Arons, B. SpeechSkimmer: A system for interactively skimming recorded speech. Proc. CHI, (1997), 3-38.

[2] Barnlund, D.C. A transactional model of communication. In Communication Theory. New Brunswick, New Jersey, 2008, 47-57.

[3] Berlo, D.K. The Process of Communication. Holt, Rinehart, \& Winston, New York, New York, USA, 1960.

[4] Cavender, A., Ladner, R., and Riskin, E. MobileASL: Intelligibility of sign language video as constrained by mobile phone technology. Proc. ASSETS, (2006).

[5] Chen, J.Y.C. and Thropp, J.E. Review of low frame rate effects on human performance. IEEE Trans. on Systems, Man, and Cybernetics - Part A: Systems and Humans 37, 6 (2007), 1063-1076.

[6] Ciaramello, F. and Hemami, S. A computational intelligibility model for assessment and compression of American sign language video. IEEE Trans. on Image Processing 20, 11 (2011).

[7] Feghali, R., Speranza, F., Wang, D., and Vincent, A. Video quality metric for bit rate control via joint adjustment of quantization and frame rate. IEEE Trans. on Broadcasting 53, 1 (2007), 441-446.

[8] Harrigan, K. The SPECIAL system: self-paced education with compressed interactive audio learning. Journal of Research on Computing in Education 3, 27 (1995), 361-370.

[9] Heiman, G.W. and Tweney, R.D. Intelligibility and comprehension of time compressed sign language narratives. Journal of Psycholinguistic Research 10, 1 (1981), 3-15.

[10] Holm, S. A simple sequentially rejective multiple test procedure. Scandinavian Journal of Statistics 6, 2 (1979), 65-70.

[11] Hooper, S., Miller, C., Rose, S., and Veletsianos, G. The effects of digital video quality on learner comprehension in an American sign language assessment environment. Sign Language Studies 8, 1 (2007), 42-58.

[12] Johnson, B.F. and Caird, J.K. The effect of frame rate and video information redundancy on the perceptual learning of American sign language gestures. 1996. http://citeseerx.ist.psu.edu/viewdoc/summary?doi=10.1.1.16. 9464.

[13] Koul, R. Synthetic speech perception in individuals with and without disabilities. 19, 1 (2003), 49-58.

[14] Kurtnoise. Yet another MP4 box user interface for Windows users. 2009. http://yamb.unite-video.com/index.html.

[15] Masry, M. and Hemami, S.S. An analysis of subjective quality in low bit rate video. International Conference on Image Processing, IEEE (2001), 465-468.

[16] McCarthy, J., Sasse, M.A., and Miras, D. Sharp or Smooth? Comparing the effects of quantization vs. frame rate for streamed video. Proc. CHI, (2004).
[17] Nemethova, A., Ries, M., Zavodsky, M., and Rupp, M. PSNR-based estimation of subjective time-variant video quality for mobiles. Measurement of Audio and Video Quality in Networks, (2006).

[18] Omoigui, N., He, L., Gupta, A., Grudin, J., and Sanocki, E. Time-compression. Proc. CHI, ACM Press (1999), 136-143.

[19] Ou, Y., Ma, Z., and Wang, Y. A novel quality metric for compressed video considering both frame rate and quantization artifacts. Workshop on Video Processing and Quality Metrics for Consumer Electronics, (2009).

[20] Richardson, I. vocdex: H.264 tutorial white papers. 2004.

[21] Saks, A. and Hellström, G. Quality of conversation experience in sign language, lip - reading and text. ITU-T Workshop on End-to-end QoE/QoS, (2006).

[22] Shannon, C.E. A mathematical theory of communication. The Bell System Technical Journal 27, 379-426 (1948), 623 656.

[23] Sperling, G., Landy, M., Cohen, Y., and Pavel, M. Intelligible encoding of ASL image sequences at extremely low information rates. Computer Vision Graphics, and Image Processing 31, (1985), 335-391.

[24] Thu, H. and Ghanbari, M. Scope of validity of PSNR in image/video quality assessment. Electronic Letters 44, 13 (2008), 800-801.

[25] Tran, J.J., Johnshon, T., Kim, J., Rodriguez, R., Yin, S., Riskin, E., Ladner, R., and Wobbrock, J.O. A web-based user survey for evaluating power saving strategies for Deaf users of MobileASL. Proc. ASSETS, (2010), 115-122.

[26] Tran, J.J., Kim, J., Chon, J., Riskin, E., Ladner, R., and Wobbrock, J.O. Evaluating quality and comprehension of real-time sign language video on mobile phones. Proc. ASSETS, (2011), 115-122.

[27] Wang, Y. and Ou, Y. Modeling rate and perceptual quality of scalable video as functions of quantization and frame rate and its application in scalable video adaptation. IEEE Trans. on Circuits and Systems for Video Technology, (2012), 671682.

[28] Wang, Z., Bovik, A., and Lu, L. Why is image quality assessment so difficult? IEEE Acoustic, Speech, and Signal Processing, (2002), 3313-3316.

[29] Wiegang, T., Schwarz, H., Joch, A., Kossentini, F., and Sullivan, G. Rate-constrained coder control and comparison of video coding standards. IEEE Trans. Circuits Systems Video Technology 13, 7 (2003), 688-703.

[30] Winkler, S. and Mohandas, P. The evolution of video quality measurement: from PSNR to hybrid metrics. IEEE Trans. on Broadcasting 54, 3 (2008), 660-668.

[31] Wobbrock, J.O., Findlater, L., Gergie, D., and Higgins, J.J. The Aligned Rank Transform for nonparametric factorial analyses using only ANOVA procedures. Proc. CHI, (2011), 143-146.

[32] Yadavalli, G., Hemami, S., and Masry, M. Frame rate preferences in low bit rate video. IEEE Image Processing, (2003), 441-444.

[33] Yang, K., Guest, C., El-Maleh, K., and Das, P. Perceptual temporal quality metric for compressed video. IEEE Trans. on Multimedia 9, (2007), 1528-1535.

[34] Mobile growth driving out unlimited data. http://www.pcworld.com/businesscenter/article/242376/mobi le_growth_driving_out_unlimited_data.html.

[35] Apple - QuickTime - Download. http://www.apple.com/quicktime/download/. 